\title{
Semantic and Syntactic Forces in Noun Phrase Production
}

\author{
Gabriella Vigliocco, Marcus Lauer, Markus F. Damian, and Willem J. M. Levelt \\ Max Planck Institute for Psycholinguistics
}

\begin{abstract}
Three experiments investigated semantic and syntactic effects in the production of phrases in Dutch. Bilingual participants were presented with English nouns and were asked to produce an adjective + noun phrase in Dutch including the translation of the noun. In 2 experiments, the authors blocked items by either semantic category or grammatical gender. Participants performed the task slower when the target nouns were of the same semantic category than when they were from different categories and faster when the target nouns had the same gender than when they had different genders. In a final experiment, both manipulations were crossed. The authors replicated the results of the first 2 experiments, and no interaction was found. These findings suggest a feedforward flow of activation between lexico-semantic and lexico-syntactic information.
\end{abstract}

Speaking involves the retrieval of lexical representations that correspond to our intentions and the development of a syntactically well-formed frame for the to-be-uttered sentence. The development of such a frame is, in part, guided by syntactic information specific to each word: for example, a word's grammatical category, the subcategorization requirements of verbs, and a noun's gender (for languages such as Dutch). The focus of this article is on the relationship between retrieving a lexical representation that specifies the meaning the speaker wants to convey and retrieving the associated lexico-syntactic information.

Across languages, lexico-syntactic properties of words are often linked to lexico-semantic properties. For example, for grammatical class, the semantic distinction between objects, actions, and properties corresponds to a syntactic distinction between nouns, verbs, and adjectives. The semantic dimension of biological gender corresponds to the syntactic distinction between masculine and feminine nouns, and the semantic distinction between entities that can be counted and substances is captured by the syntactic distinction between count and mass nouns. The existence of such correspondencies between the semantic and the syntactic dimensions has been taken by some authors as indicating a conceptual foundation

Gabriella Vigliocco, Marcus Lauer, Markus F. Damian, and Willem J. M. Levelt, Max Planck Institute for Psycholinguistics, Nijmegen, the Netherlands.

Marcus Lauer is now at the Department of Cognitive Science, University of California, San Diego. Markus F. Damian is now at the Department of Experimental Psychology, University of Bristol, Bristol, United Kingdom.

While conducting this work Gabriella Vigliocco was supported by a fellowship from the Max Planck Society, by National Science Foundation Grant SBR9711829, and by Human Frontier Science Program Grant HFSP148/2000. Marcus Lauer was supported by a Fellowship from the Graduate School, University of Wisconsin-Madison.

We thank Ardi Roelofs and two anonymous reviewers for their insightful comments on previous versions of the article.

Correspondence concerning this article should be addressed to Gabriella Vigliocco, who is now at the Department of Psychology, University College London, 26 Bedford Way, London WC1H OAP, England. E-mail: g.vigliocco@ucl.ac.uk for the syntactic distinctions (e.g., Bates \& MacWhinney, 1982; Langacker, 1987). However, these correspondencies between semantic and syntactic properties are limited, to an important extent. With respect to grammatical class, actions (e.g., "to bomb") can also be denoted by nouns (e.g., bombardment). In a number of languages, gender of nouns is not only a property of nouns referring to entities but also of nouns referring to objects and abstract entities for which there is no clear semantic correlate. And, cases exist, in English for example, in which the distinction between count and mass nouns does not reflect the semantic distinction between entities that can be counted and substances (e.g., abstract nouns such as opinion and knowledge). The different degree of correspondence between lexico-semantic and lexico-syntactic properties across languages and types of lexico-syntactic properties provides us with a valuable opportunity to assess the relationship between these two types of information in language processing.

Although many researchers agree that these two types of information are separable (see Bowers, Vigliocco, StadthagenGonzales, \& Vinson, 1999, for a discussion), such a distinction has been disputed in some camps (e.g., Sera, Berge, \& del CastilloPintado, 1994). If one assumes that they are distinct types of information, an important and largely unexplored question concerns how strictly lexico-semantic and lexico-syntactic information are associated. In this study we report an initial investigation of this question in the domain of sentence production. We consider a syntactic property that does not have a clear semantic counterpart (grammatical gender in Dutch), and we experimentally assess whether manipulations of grammatical gender and manipulations of lexico-semantic information interact. In this article we will assume that syntactic information that specifies how to use individual words in sentences, such as grammatical class or grammatical gender (in languages such as Dutch) is lexically specified, along the lines proposed by Levelt (1989); McDonald, Pearlmutter, and Seidenberg (1994); and Vosse and Kempen (2000). Specifically, with respect to grammatical gender, we assume that an abstract noun-phrase frame that specifies the gender of a noun and the other words that can be used in the phrase is lexically stored and accessed via the lexical representation for the noun. 
The Retrieval of Lexico-Semantic Representations and of Lexico-Syntactic Information in Sentence Production

Retrieving words for speaking entails, at least, the retrieval of lexico-semantic, lexico-syntactic, and word-form information. Theories of sentence production generally agree that lexical retrieval during production proceeds in two main steps: meaningbased retrieval and form-based retrieval (Butterworth, 1989; Dell, 1986; Foygel \& Dell, 2000; Fromkin, 1971; Garrett, 1975; Levelt, 1989; Levelt, Roelofs, \& Meyer, 1999). Evidence compatible with this separation comes from a variety of sources (see Vigliocco, Vinson, Martin, \& Garrett, 1999, for an overview), including studies of slips of the tongue and of the tip-of-the-tongue phenomenon (e.g., Dell, 1986; Fromkin, 1971; Garrett, 1975, 1980; Vigliocco, Antonini, \& Garrett, 1997), chronometric experiments using the picture-word interference paradigm (e.g., Schriefers, Meyer, \& Levelt, 1990), and neuropsychological investigations (e.g., Buckingham, 1979; Goodglass, Kaplan, Weintraub, \& Ackerman, 1976; Vigliocco et al., 1999). It is also agreed on that lexico-semantic and lexico-syntactic information are separately represented. Furthermore, it is generally assumed that both lexicosemantic and lexico-syntactic information are available to the production system before the phonological form, because both are necessary for grammatical encoding, that is, for developing a syntactically well-formed frame for a sentence (e.g., Levelt et al., 1999; van Turennout, Hagoort, \& Brown, 1998). ${ }^{1}$ Such a syntactically specified frame would represent both open-class (e.g., nouns, verbs, etc.) and closed-class words (e.g., determiners, pronouns, inflections - that is, syntactic markers) in an abstract, not phonologically specified format. Evidence compatible with the abstract nature of this level of representation comes from, for example, observations of slips of the tongue. Slips occurring at this level, such as exchanges (Example 1, below) and semantically related word substitutions (Example 2, below) are to some important extent not influenced by the phonological make up of the words but only by their syntactic properties for exchanges or their semantic and syntactic properties for substitutions (Garrett, 1980, 1982).

(1) Well you can cut rain in the trees [intended: trees in the rain; Garrett, 1982, p. 49]

(2) It's a far cry from the twenty-five dollar days [intended: cent; Garrett, 1982, p. 52]

How is the flow of information regulated? In WEAVER ${ }^{++}$ (Levelt et al., 1999), a lexical concept is activated on the basis of the speaker's intentions. Activation from the lexical concept spreads both to other concepts semantically related to the target and to the corresponding abstract lexical representations (referred to as lemmas) via two-way connections. Lemmas corresponding to activated concepts will compete for selection. In this theory, lemmas are conceived of as intermediate representations both between lexical concepts and word-form representations and between lexical concepts and syntactic properties. Syntactic properties such as gender or grammatical class would be retrieved during grammatical encoding (i.e., when lemmas are retrieved) to allow for the building of a syntactic frame for the to-be-uttered sentence. Their retrieval is assumed to be dependent on the selection of the corresponding lemma, although activation would spread automat- ically from lemmas to lexico-syntactic properties before lemma selection. Active syntactic frames, however, would not feed back activation to the lexical selection process. Word-form retrieval would also be strictly contingent on lemma selection, and with respect to this interface no spreading of activation from lemmas to word forms nor feedback from word forms to lemmas would be allowed. Hence, in this theory, retrieving lexico-semantic representations and their corresponding syntactic features is a sequential process, for which activation flow is strictly feedforward-that is, the frame that is built on the basis of the lexical syntactic properties cannot affect the process of lemma selection. Note, however, that in contrast to the two-step retrieval process of lexico-semantic and word-form information, activation is allowed to spread (i.e., cascade) from lexico-semantic to lexico-syntactic information.

In the model developed by Dell (1986) and Dell, Schwartz, Martin, Saffran, and Gagnon (1997), as well as in the proposal by Stemberger (1985), activation is instead assumed to spread bidirectionally among lexico-semantic, word-form, and lexicosyntactic information. With respect to the interface between lexico-semantic and word-form information, for instance, we have that on the basis of the speaker's intention activation spreads over a constellation of semantic features and to their corresponding abstract lexical representations, including the target. Some activation will also cascade to the corresponding phonological codes. Because of feedback, then, the selection of a given abstract lexical representation is influenced not only by the semantic properties of the other lexical candidates that share semantic features with the target but also by their phonological properties.

With respect to the interface between lexico-semantic and lexico-syntactic information, interactive activation models are quite underspecified and clear predictions may strictly depend on details of parameter setting in the models. However, in parallel to the interface between lexico-semantic and word-form retrieval, a conceivable scenario is as follows. The cascading nature of the process allows for the activation (or generation) of syntactic frames for highly activated, but not yet selected, lexico-semantic representations. Feedback from the frame to the lexical retrieval process could entail a biasing effect from the syntactic specification of the frame to lexical selection. Stemberger (1985) discussed this latter assumption with respect to grammatical class. He argued that if a syntactic frame for a noun phrase, for example, is under construction, such a frame may bias the lexical selection process, enhancing the activation of those entries that can fit into the frame (i.e., other nouns). Hence, in this specific scenario, the semantically driven lexical retrieval process may be affected not only by the word-form properties (see Dell, 1986; Levelt, 1999, for a discussion) but also by the syntactic properties of highly activated entries.

\footnotetext{
${ }^{1}$ An alternative view has been recently proposed by Caramazza and colleagues (Caramazza 1997; Caramazza \& Miozzo, 1997; Miozzo \& Caramazza, 1997), according to which retrieving syntactic information does not necessarily occur prior to the retrieval of phonological information about the word (see Vigliocco et al., 1999, for a discussion). Note that with respect to the interface between lexico-semantics and lexico-syntactic information this alternative view would predict a strong separation between the two types of information.
} 
A substantial number of studies have addressed whether the retrieval of lexico-semantic and word-form information proceeds in a strict serial manner or whether they interact (see Levelt et al., 1999 for a review). These studies provide evidence for the sequentiality of the process and indicate that at least a limited amount of cascading of activation (maybe as limited as applying to synonyms only) needs to be granted. The question concerning whether feedback from word forms to lexico-semantic representations needs to be incorporated is still a matter of dispute.

Much less is known with respect to how the retrieval of lexicosemantic and lexico-syntactic information is orchestrated in production, and existing theories of lexical retrieval are somewhat underspecified in this respect. However, in parallel to the discussion concerning the interface between semantically driven and form-driven retrieval, one can distinguish between (a) theories that assume that building a syntactic frame for a sentence is a process that occurs only after the semantically driven lexical retrieval is completed (hence the properties of the syntactic frame cannot affect the semantically driven retrieval process) and (b) views in which the semantically driven lexical retrieval and phrasal retrieval are interactive processes (in which phrasal retrieval can affect the outcome of the lexico-semantic retrieval process via feedback). Below we review the existing literature concerning this interface.

Speech-error data provide evidence that is compatible with a strict association between semantically driven lexical retrieval and the retrieval of phrasal information. Relevant to our discussion here are semantically related word-substitution errors (e.g., Fromkin, 1971), such as in Example 2 above, in English, and Examples 3 and 4, in Dutch and Italian, respectively.

(3) roer even daarin tot de saus... kaas gesmolten is [stir that for a while until the sauce... has melted] [intended: cheese has melted]; Schelvis (1985)

(4) Volevo togliere un altro foglio [I wanted to take off another sheet] [intended: put on]; Vigliocco (2001)

It is generally agreed that these errors arise in the mapping between conceptual and lexico-semantic representations (e.g., Garrett, 1982). On the basis of the conceptual specification, the target lexico-semantic representation and a set of semantically related competitors are activated and, in error-free production, the target is selected from among the semantic competitors. From time to time, however, a semantic competitor may become more strongly activated than the target, and therefore is erroneously selected, as in the examples above.

These errors are constrained by the lexico-syntactic properties of the target. For example, the target word and the intruding word are always of the same grammatical class (Garrett, 1980) - that is, a noun is replaced by another noun, a verb by another verb, and so forth. Such a grammatical class constraint is compatible with an interactive view according to which a highly activated syntactic frame can exert a biasing effect, via feedback, on lexical selection (and in fact, the existence of a grammatical category constraint on the semantically motivated errors is used by Stemberger, 1985, to motivate interactivity between lexical retrieval and phrasal construction). However, because the grammatical class constraint has been observed in spontaneously occurring errors, there may be alternative accounts. In particular, one cannot exclude that the targets represented in the corpora have intruders that share grammatical class as the closest semantic neighbors. As we outlined above, there is a large degree of transparency between the semantic properties of a word and its grammatical class. Therefore it may be the case that semantic similarity alone may account for the grammatical class constraint in spontaneously occurring semantic substitution errors.

A more compelling case is the observation by Berg (1992) and Marx (1999) that in German, targets and semantically related intruders are of the same gender more often than chance would predict. Two examples of such errors (from Marx, 1999, p. 608), preserving the gender of the target noun, are reported in Examples 5 and 6 below.

(5) ... wo das (neut.) Wörterbuch (neut.), äh Lexikon (neut.), äh Vokabelheft (neut.) ist [... where the dictionary, uh encyclopedia, uh vocabulary notebook is]

(6) ... wenn du gleich Zitronen (fem.), Apfelsinen (fem.) auspreBt. [... when you right away lemons, oranges squeeze]

Because gender in German is a property that, for nouns referring to objects and abstract entities, does not bear conceptual force, such a finding is difficult to account for in terms of greater semantic similarity among words of the same grammatical gender, unless one subscribes to a view, as proposed by Sera et al. (1994), according to which arbitrary syntactic properties also can become part of the semantic specification of a word after they have been learned (see General Discussion). They are also prima facie difficult to account for by a strictly serial model such as WEAVER ${ }^{++}$. These results, instead, are easily accounted for by interactiveactivation models as a biasing effect (via feedback) from the syntactic frame to the lexical selection process.

Prior chronometric studies of lexical retrieval during production provide evidence that suggests a different time course for the retrieval of lexico-semantic and lexico-syntactic information. However, these data are silent with respect to how the retrieval of lexico-semantic and lexico-syntactic information is orchestrated. Most studies have used a picture-word interference paradigm in which speakers are presented with target pictures to name while at different stimulus onset asynchronies (SOAs; e.g., immediately prior to the picture presentation or simultaneous to the picture presentation). Words semantically related or unrelated with the target picture are also presented. With respect to lexical selection, a large number of studies, since Schriefers et al.'s (1990) study, have established that such a process can be characterized in terms of competitive retrieval. The crucial finding, for the purposes of our study, is that when the picture is immediately preceded by a semantically related word, speakers are slower in producing the target label than when the word and the picture are unrelated. This finding has been interpreted in terms of lexical competition as follows: The semantically related word enhances the activation of one of the lexical competitors, rendering the selection process harder, resulting in longer naming latencies.

With respect to syntactic properties, a number of studies have also used the picture-word interference paradigm to investigate whether the retrieval of a given syntactic feature, and hence the construction of a given syntactic frame to describe a picture, is influenced by the presentation of a word (again at different SOAs) that shares or does not share syntactic properties. One syntactic 
property that has been addressed is the grammatical gender of nouns. Studies have examined different languages, including Dutch (Schriefers, 1993), German (Schriefers \& Teruel, 2000), and Italian (Miozzo \& Caramazza, 1999). Schriefers (1993) and Schriefers and Teruel (2000) reported that speakers of Dutch and German were faster in producing phrases with gender-marked elements (determiners or adjectives) when the picture to be described and the interfering word shared the same gender than when they did not at SOAs later than those at which a semantic effect was observed. Such a gender-congruency effect may arise because the activation of a given gender (even for the distracting word, for which a phrase is not produced) is enhanced by the simultaneous presentation of another word sharing the same gender, and therefore the word retrieval would be faster than it would be when the two lexical entries do not share the same gender. Note that although such an account is based on the assumption of facilitation when the two words share the same properties, such a conclusion is not warranted because the experiments lack a neutral (no gender) baseline. Furthermore, although not directly relevant to the purpose of our study, it is worth noting here that, at least when determiner + noun phrases are considered, cross-linguistic differences have been reported. Although an effect of gender congruency has been shown in languages such as Dutch and German, this effect has not been replicated in a language such as Italian (Miozzo \& Caramazza, 1999). A possible explanation for this difference (Miozzo \& Caramazza, 1999) may be that although the phonological form of the determiner is only dependent on the gender of the noun in Dutch and German, it is also dependent on the phonological word onset in Italian; hence in this latter language even if the syntactic feature might be retrieved before the phonological encoding of the phrase, the determiner cannot be realized until the segmental content of the noun is encoded. In German and Dutch, instead, the phonological encoding of the determiner does not need to wait until the segmental onset of the noun is spelled out.

In these studies, the manipulation of gender congruency was combined with the manipulation of semantic relatedness. To summarize the main results, across the two languages for which a gender congruency effect was found (Dutch and German) and across different types of phrases produced (determiner + noun; adjective + noun; and determiner + adjective + noun) to describe the target picture, a semantic-relatedness effect was found at the more negative SOAs (except Schriefers, 1993, Experiment 1) and a gender-congruency effect was found at later SOAs. These two factors were also found to interact (or at least to show a trend toward a significant interaction) for intermediate SOAs between the main effect of semantic relatedness and the main effect of gender congruency. That is, at these intermediate SOAs, a gendercongruency effect was observed for semantically related trials only. What can one conclude from these results? First, the finding of a semantic-relatedness effect followed by a gender-congruency effect is consistent with the hypothesis of the separation between these two types of information and with their distinct time course. The reported interaction between the two factors is more difficult to interpret and does not directly bear on the issue of whether the semantically driven lexical retrieval process and the process of building syntactic frames interact. This is because, at those SOAs, the interaction was due to the fact that the gender congruency effect was observed only for semantically related target-distractor pairs and not for semantically unrelated pairs. Because the seman- tically related condition is the one in which speakers are slower, the observed interaction may simply reflect the fact that the gender-congruency effect required additional time to arise.

To summarize, two lines of studies have so far provided some information concerning how the retrieval of lexico-semantic and lexico-syntactic information is orchestrated in time. The existence of syntactic constraints on semantically related substitution errors suggests that activated frames for competing words may affect the lexical selection process via feedback. Picture-word interference studies, instead provide us only with an indication that the lexical selection process precedes in time the retrieval of lexico-syntactic information.

\section{Overview of the Experiments}

In a series of three experiments in which Dutch speakers were asked to produce adjective + noun phrases, we assessed whether lexical selection and the retrieval of a syntactic frame produced interactive effects. In our study, we introduced a new methodology to investigate conjointly semantic and syntactic effects in phrase production. The aim of our first two experiments was to establish whether our new methodology allowed us to observe effects of semantic relatedness and of syntactic congruency in isolation. In our third experiment we orthogonally combined the semantic and the syntactic manipulations.

In the experiments we induced context effects, either semantic or syntactic (gender). Our methodology borrowed from studies that have used implicit priming (context effects induced by the previous production of a word, to investigate phonological encoding in production; e.g., Meyer, 1990) and structural priming (to investigate the effects of repeating a syntactic structure; e.g., Bock, 1986). With respect to semantics, a basic prediction was that if speakers produce phrases in the context of producing other phrases with semantically related words (semantically homogeneous), the speakers will be slower than when they are producing the same phrases but in the context of producing other phrases with semantically unrelated words (semantically heterogeneous). This is because in the former case the lexical candidates in the response set act as competitors being highly activated in virtue of their semantic relatedness, whereas in the latter case for each trial the activation level of the nontarget lexical candidates is not as high, because they are not semantically related. With respect to lexico-syntactic properties, we predicted that speakers should be faster in producing phrases that share the same syntactic properties (syntactically homogeneous) than in producing phrases that do not (syntactically heterogeneous). This prediction derived from the assumption that having already prepared an abstract syntactic frame (e.g., Bock, 1986) or having already enhanced the activation of a specific syntactic feature (Levelt et al., 1999), the encoding of a following phrase that shares the same structure can take advantage of the residual activation from the previous trial (see also Hartsuiker \& Kolk, 1998; Pickering \& Branigan, 1999). It is important to note here that we assumed that sharing the same syntactic frame is advantageous. However, as indicated above, on the basis of the picture-word interference studies, it is not clear whether indeed the effect observed is facilitatory or inhibitory because of the lack of a neutral baseline. Our assumption was based on the fact that the task we used here, in which speakers produce one phrase after the other, was in continuity with the paradigm used in syntactic 
priming studies in which the previous production of a given structure increased the preference for reusing the same structure as compared with a neutral baseline. On a related note, the models introduced above do not allow for interpreting syntactic effects as inhibitory because they do not incorporate inhibitory links between the different syntactic features (for examples of models in the domain of sentence comprehension that embed inhibitory links between lexico-syntactic properties, see McDonald et al., 1994, and Vosse \& Kempen, 2000; see also Ferreira, 1996, for some evidence against syntactic inhibition in production).

Crucially, if we were to observe these two effects in isolation (Experiments 1 and 2) then we could cross them (Experiment 3 ) to assess whether the two factors are additive or whether they interact in a way that can be interpreted as a biasing effect from the syntactic frame to the lexical retrieval process. On the basis of the described syntactic constraints on semantically related substitution errors, such a biasing effect could be found in our study. In particular, if activation would feed back from a frame that is highly activated (because it is reused, as in the syntactically homogeneous condition) to the semantically driven retrieval process, it might enhance the activation of those lexical candidates that share the same syntactic feature. These lexical candidates included the target word and other words in the response set. We predicted that in the condition in which the words in the response set are semantically related (semantically homogeneous), the semantic competitors would be already highly activated in virtue of their meaning relationship with the target. If they also shared syntactic features with the target, their activation would be further increased by the feedback, increasing the competition, and therefore increasing the resolution time. Hence, the advantage of sharing the same syntactic structure could be reduced or even canceled in the semantically homogeneous condition. That is, we may find an effect of semantic and syntactic context and an interaction between the two such that reaction times in the semantically and syntactically homogeneous condition are the same or longer than in the semantically heterogeneous but syntactically homogeneous condition. Note here that such prediction was not directly derived by any specific interactive activation model proposed thus far. It derived as an extension of a feedback-based account of the syntactic constraints arising in semantically related substitution errors. We felt that if the postulated feedback was strong enough to bias lexico-semantic retrieval toward competitors sharing the same syntactic features (hence resulting in a substitution error in which target and intruder share the same syntactic properties), it should also be strong enough to affect the time course of lexico-semantic retrieval processes. Strictly serial models such as WEAVER ${ }^{++}$would not predict such an interaction.

We used a translation task (from L2: English to L1: Dutch), instead of a picture-naming task, to allow us higher degrees of freedom in choosing materials for the experiments. Studies have established that such a task taps into lexico-semantic knowledge, and this task has been already successfully used to address questions concerning lexical retrieval in production (la Heij et al., 1990). In particular, we used a modified version of the blocking paradigm introduced by Kroll and Stewart (1994), in which bilingual speakers are presented with blocks of words that are semantically homogeneous (i.e., all from the same semantic category) or semantically heterogeneous (i.e., from different semantic categories) and are asked to produce the corresponding word in the other language. For single-word production, naming latencies for words from homogeneous blocks were slower than for heterogeneous blocks (the lexical competition effect; see also Damian, Vigliocco, \& Levelt, 2001). We modified this task by asking speakers of Dutch to produce a noun phrase consisting of the Dutch equivalent of big + noun or small + noun in response to English words written in a big or small font. The required adjective was gender marked in Dutch according to the common or neuter gender of the nouns. Grammatical gender in Dutch (common or neuter) is not conceptually motivated, hence, in our investigation we assessed whether a syntactic property that is only arbitrarily linked to the meaning of words affects lexico-semantic processes.

\section{Experiment 1}

The first experiment tested for a semantic effect. The target nouns were blocked by semantic category but not by gender. Some blocks contained six words of the same category (semantically homogeneous blocks), whereas the other blocks contained words from different semantic categories (semantically heterogeneous blocks). We predicted that latencies to produce adjective + noun would be slower for semantically homogeneous blocks than for semantically heterogeneous blocks.

\section{Method}

Participants. Twelve Dutch native speakers from the participants pool of the Max Planck Institute for Psycholinguistics participated in this experiment and were each paid Hfl. 8.50 (approximately U.S. \$4). All participants were explicitly required to have good knowledge of English. The last 5 participants filled out questionnaires regarding their English skills. On a scale of 0 (no knowledge of English) to 7 (equally fluent in Dutch and English), participants' mean rating for their knowledge of English was $4.8(S D=0.7)$. The mean age at which these participants began to learn English was 9.8 years (range $=6-13$ ).

Materials. Six nouns from each of six semantic categories (animals, body parts, clothing, plants, vehicles, and weapons) that were to be translated from English to Dutch were used in this experiment. The English prompt words had an average word-form frequency of 87 per million in the CELEX (Baayen, Piepenbrock, \& Gulikers, 1995) database, whereas the Dutch response words had an average CELEX frequency of 86 per million. Three of the Dutch nouns in each category were of the common gender and three were of the neuter gender. The experimental task required that participants produce an adjective + noun phrase in which the adjective was the Dutch word klein [small] or groot [big]. All of these nouns were chosen so that it was pragmatically adequate for them to be used with the adjectives big and small in Dutch.

Some evidence suggests that cognates are translated in a different manner than noncognates (de Groot, Dannenburg, \& van Hell, 1994; but see Kroll \& Stewart, 1994). Specifically, some researchers argue that cognates may be translated using direct word-to-word associations between the speaker's two languages, bypassing lexico-semantics. To avoid any problems that might arise from this complication, Dutch-English cognates were avoided wherever possible in the selection of the stimuli. See Appendix A for a complete list of the words used in Experiment 1.

These stimuli were arranged in a $6 \times 6$ matrix such that the rows corresponded to the semantic categories. From this matrix, 12 lists of six items were derived, corresponding to the rows (in which the six items were from a single category-homogeneous condition) and columns (in which the six items were from six different categories-heterogeneous condition). Thus, the same items served as stimuli in both the homogeneous and heterogeneous conditions, but they were presented within a homogeneous 
or heterogeneous semantic context. Items were permutated such that each list included three common-gender words and three neuter-gender words.

From these 12 lists, experimental blocks were generated in which each of the six English prompt words was presented eight times, for a total of 48 trials per block. Words appeared in either large or small print (see Apparatus), and each word was presented four times in each size in one block. The presentation of stimuli was pseudorandomized such that a word was never repeated on consecutive trials and no more than three words of the same print size appeared consecutively.

Design. The experimental design included semantic context (heterogeneous vs. homogeneous) as a within-subjects variable.

Apparatus. Participants were tested in a sound-attenuating booth. Stimuli were presented on an IBM-compatible computer. They were shown in white on a black background in either large font (64-point Arial) or small font (32-point Arial). Reaction times for the generation of the Dutch translations were measured using a voice-activated relay to the nearest millisecond.

Procedure. Participants were tested individually. Each participant was tested on each of the 12 experimental blocks. They were instructed to respond to the English target word by producing an adjective + noun phrase in Dutch in which the adjective described the size of the word (groot for words in large print, klein for words in small print) and was inflected for the gender of the Dutch noun (grotelkleine for common gender, groot/klein for neuter gender), whereas the noun was the Dutch translation of the English prompt word. See Figure 1 for a schema of this experimental manipulation.

At the beginning of a particular block, participants were shown all of the six English nouns occurring in that block, together with their Dutch translation, and were given as much time as they needed to look at the list. Once the participants indicated that they were ready, the experimental block began. Experimental blocks were presented in an alternating sequence of heterogeneous and homogeneous blocks such that half of the participants started with a heterogeneous block and the other half with a homogeneous block. The order in which the blocks were presented was determined by a sequentially balanced Latin square design.

At the beginning of each individual trial, a fixation cross appeared at the center of the screen for $500 \mathrm{~ms}$, followed by a blank period for $400 \mathrm{~ms}$. Then the prompt word appeared at the center of the screen for 1,500 ms. Response latencies were measured relative to the prompt onset. Following each response, the experimenter judged the response to be either correct or incorrect. The incorrect responses included the generation of the wrong adjective, wrong noun, or wrong inflection on the adjective; when the voice key triggered to a sound other than a proper response such as clicking, smacking, or other lip movement sounds; the word een [a/an] or $u h$ at the start of a response; unclear responses; or hardware malfunctions. Each trial was followed by a 1,100-ms intertrial interval.

The entire experiment consisted of 576 trials and lasted approximately $45 \mathrm{~min}$.

\section{Participant sees:}

\section{Participant responds:}

$\begin{array}{ll}\text { chicken } & \text { "grote kip" [big-c chicken] } \\ \text { chicken } & \text { "kleine kip" [small-c chicken] } \\ \text { horse } & \text { "groot paard" [big-n horse] } \\ \text { horse } & \text { "klein paard" [small-n horse] }\end{array}$

Figure 1. Schema of experimental manipulation.

\section{Results and Discussion}

Responses judged to be incorrect by the experimenter, as well as response times longer than $1,500 \mathrm{~ms}$ or shorter that $250 \mathrm{~ms}$, were removed from the response-time analysis (7.8\%). As the difference between homogeneous and heterogeneous blocks was one of semantic context, which may require several trials to become established, responses to the first presentation of each prompt word of both large and small size in each block were also removed from the analysis.

Table 1 displays the mean response latencies and error percentages in the two experimental conditions, indicating substantially longer response latencies $(740 \mathrm{~ms})$ as well as higher error percentages $(8.6 \%)$ in the homogeneous than in the heterogeneous (707 ms and $5.9 \%$ ) condition.

An analysis of variance (ANOVA) that included semantic context as a within-subjects variable indicated a significant effect in the response latencies, $F_{1}(1,11)=44.11, M S E=6,438, p<.001$; $F_{2}(1,35)=32.47, M S E=23,117, p<.001$, and in the errors, $F_{1}(1,11)=10.60, M S E=43.76, p=.008 ; F_{2}(1,35)=8.02$, $M S E=131.28, p=.008$. Thus, a semantically homogeneous context exerted an interference effect on responses. ${ }^{2}$

Speakers performed this task more slowly on blocks in which the target words were semantically related. This finding can be accounted for by assuming that in the mapping between conceptual and lexical knowledge, semantically related lexical representations are highly activated and act as competitors in the retrieval process. It is important to note here that the semantic-context effect we reported is specific to lexical processes, and it is not more generally a conceptual effect. In another series of studies using the same paradigm we adopted here and presenting pictures to be named as a single word, Damian et al. (2001) found that the semanticinterference effect was present when participants were asked to name the pictures, however, the interference effect disappeared when participants were asked to perform a conceptual task that did not require lexicalization.

\section{Experiment 2}

The second experiment tested for gender congruency effects using our new paradigm. Blocks of English words with the same gender in Dutch (gender homogeneous) and blocks of words with different gender (gender heterogeneous) were presented to speakers who were asked to produce, again, an adjective + noun phrase, as in Experiment 1. In contrast to Experiment 1, all blocks were semantically heterogeneous. We predicted that latencies to produce phrases in the gender-homogeneous condition should be faster than in the gender-heterogeneous condition.

\section{Method}

Participants. Twelve Dutch native speakers from the participants pool of the Max Planck Institute for Psycholinguistics, none of whom had

\footnotetext{
${ }^{2}$ An additional analysis included the factor size (small vs. large). In this and each of the following experiments, this factor showed a significant effect such that participants responded slower to large than to small prompt words. However, the factor never interacted with any of the critical variables and it is therefore not reported.
} 
Table 1

Experiments 1, 2, and 3: Response Latencies (RL; in $m s)$ and Error Percentages (EP) as a Function of Semantic (Exp. 1), Syntactic (Exp. 2), and Semantic and Syntactic (Exp. 3) Context

\begin{tabular}{|c|c|c|c|}
\hline & \multicolumn{2}{|c|}{ Semantic context } & \multirow[b]{2}{*}{ Effect } \\
\hline & Heterogeneous & Homogeneous & \\
\hline $\begin{array}{l}\text { RL } \\
\text { EP }\end{array}$ & $\begin{array}{c}707(34) \\
5.9\end{array}$ & $\begin{array}{c}740(37) \\
8.6\end{array}$ & $\begin{array}{l}-33 \\
-2.7\end{array}$ \\
\hline \multicolumn{4}{|c|}{ Experiment 2} \\
\hline & \multicolumn{2}{|c|}{ Syntactic context } & \\
\hline & Heterogeneous & Homogeneous & Effect \\
\hline RL & $793(31)$ & $777(30)$ & 16 \\
\hline \multirow[t]{3}{*}{ EP } & 8.1 & 8.0 & 0.1 \\
\hline & \multicolumn{2}{|c|}{ Experiment 3} & \\
\hline & \multicolumn{2}{|c|}{ Semantic context } & \\
\hline Syntactic context & Heterogeneous & Homogeneous & Effect \\
\hline \multicolumn{4}{|l|}{ Heterogeneous } \\
\hline RL & $650(25)$ & $665(28)$ & -15 \\
\hline $\mathrm{EP}$ & 6.4 & 8.4 & -2.0 \\
\hline \multicolumn{4}{|l|}{ Homogeneous } \\
\hline RL & $632(24)$ & $648(26)$ & -16 \\
\hline EP & 6.3 & 6.8 & -0.5 \\
\hline
\end{tabular}

Note. Standard errors of the mean are in parentheses.

participated in Experiment 1, took part in this experiment and were each paid Hfl. 8.50 (approximately U.S. \$4). All participants were explicitly required to have good knowledge of English. All 12 participants filled out questionnaires regarding their level of English skills. On a scale of 0 (no knowledge of English) to 7 (equally fluent in Dutch and English), the mean level of participants' self-reported English knowledge was $4.8(S D=0.9)$. The mean age at which these participants began to learn English was 9.0 years (range $=6-12$ ).

Materials. The same six categories with six words per category as in Experiment 1 were used (see Appendix A). However, this time, all 12 experimental lists were semantically heterogeneous, and instead, syntactic homogeneity was manipulated such that in 6 of the lists, all response words were either common-gender or neuter-gender words (syntactically homogeneous). In the other 6 lists, half of the response words were commongender words and half were neuter-gender (het) words (i.e., syntactically heterogeneous). Experimental blocks were again generated such that each prompt word from a list was presented and translated eight times in pseudorandom order for a total of 48 trials per block. Target words appeared in either large or small print, and each word was presented four times in each size in one block.

The same practice list was used as in Experiment 1, consisting of six words not used elsewhere in the study that were gender-heterogeneous and from various semantic categories.

Design. The experimental design included syntactic context (heterogeneous vs. homogeneous) as a within-subjects variable.

Apparatus and procedure. These were identical to those used in Experiment 1.

\section{Results and Discussion}

Responses judged to be incorrect by the experimenter, as well as response times longer than $1,500 \mathrm{~ms}$ or shorter that $250 \mathrm{~ms}$ were removed from the response time analysis (8.4\%). Again, responses to the first presentation of each stimulus in each block were removed from the analysis.

Table 1 displays the mean response latencies and error percentages in the two experimental conditions, indicating faster response latencies in the homogeneous $(777 \mathrm{~ms})$ than in the heterogeneous (793 ms) condition but no effect on the error percentages $(8.0 \%$ and $8.1 \%$ in the homogeneous and heterogeneous conditions, respectively). An ANOVA that included syntactic context as a within-subjects variable indicated a significant effect in the response latencies, $F_{1}(1,11)=5.04, M S E=1,590, p=.046 ; F_{2}(1$, $35)=13.92, M S E=3,413, p=.001$, but no such effect in the errors $\left(F_{1}\right.$ and $\left.F_{2}<1\right)$. Thus, a syntactically homogeneous context exerted a facilitatory influence that was visible only in the response latencies. $^{3}$

Participants were faster when producing phrases of the same gender than when producing phrases of different gender. This result can be accounted for by theories of lexical retrieval in language production by assuming that reaccessing the same syntactic feature leads to an advantage.

The results from this experiment parallel previous studies that found gender congruency effects using a picture-word interference task (Schriefers, 1993; Schriefers \& Teruel, 2000). This effect can be interpreted in terms of enhanced activation or availability of a syntactic frame that has been just previously used. Our results are also in line with previous work concerning syntactic priming (Bock, 1986; Hartsuiker \& Kolk, 1998; Pickering \& Branigan, 1999). In syntactic priming studies it is generally observed that previously produced syntactic frames are likely to be reused in subsequent productions. One interpretation of this preference for reusing the same syntactic structure has been in terms of residual activation of a syntactic frame along the lines as discussed above. In the domain of phrasal (instead of clausal) production, our study therefore presents some first-latency data in continuity with studies that have addressed the issue of syntactic persistency in terms of structural preferences.

Because of the paradigm we used, however, there are alternative explanations for the syntactic-congruency effect we found that need to be addressed. First, because words of the same gender were presented in blocks, one may argue that the facilitatory effect in the gender-homogeneous condition came about because speakers consciously realized that all phrases in a gender-homogeneous block were of the same gender and that they used this knowledge in the preparation of the phrases. In our experiment, speakers could not prepare to articulate the first word (the adjective) even when they knew the gender because the use of one adjective or the other was related to the size (big or small) of the text. However, there may still be ways in which consciously knowing that all the words in a block are of the same gender might have helped. We asked all

\footnotetext{
${ }^{3}$ Note here that also in spontaneously occurring errors, cases of withinphrase gender mismatch between the determiner and the noun, or between an adjective and a noun, are virtually absent in gender-marked languages such as German (Marx, 1999) and Spanish (Del Viso, Igoa, \& GarciaAlbea, 1987).
} 
participants at the end of the experiment whether they noticed any of the experimental manipulations-in particular whether they noticed that words of the same or different gender were presented in blocks. In the analyses reported above, the data of only those participants who failed to notice the blocking of gender were included. Therefore, we can exclude to some extent the possibility that the observed facilitation was due to their use of a conscious strategy related to both their knowing that all words in a block were of the same gender and their knowing which gender, specifically.

Second, besides the use of conscious strategies, the facilitatory effect in the gender-homogeneous condition might have arisen because of priming at the word-form level. First, adjectives in the neuter gender end with the same inflection, "-e," hence there might have been priming from one trial to the next in this condition. However, previous work using an implicit-priming paradigm (which is similar to the paradigm used here in some important extents) has failed to show any priming for words with form overlap in noninitial positions (Meyer, 1990). This argues against the possibility of interpreting the gender facilitation in terms of word-final priming for the gender-neuter words. Second, whereas in the gender-heterogeneous condition the adjectives were both mono- and bisyllabic (klein, kleine, groot, grote), in the gender homogeneous condition they were all either monosyllabic (klein, groot) for common-gender words or bisyllabic (kleine, grote) for neuter-gender words. Therefore, one may argue that the facilitatory effect we found is not related to the gender manipulation per se, but can be accounted for as an implicit priming of the syllabic structure even in the absence of segmental overlap. However, again, work by Roelofs and Meyer (1998) speaks against such a possibility. In one experiment, they assessed, by using an implicitpriming paradigm, whether blocks of words sharing the same syllabic structure but no segmental overlap were produced faster than blocks of words that did not share the syllabic structure, and they failed to observe any effect of such manipulation. Therefore, it is unlikely that homogeneity in the number of syllables in our experiment can account for the facilitation we observed.

Finally, the syntactic effect might have arisen as repetition priming. In the syntactically homogeneous blocks, on average, a given phrase is preceded by another phrase that starts with the exact same word (e.g., they produced grote kip [big chicken] and then grote hond [big dog] on half of the trials). In the syntactically heterogeneous blocks, instead, a phrase was preceded by another phrase starting with the exact same word only one fourth of the trials, on average. Hence, the homogeneous condition presents itself as a candidate for repetition priming from one trial to the next. To address this alternative account, we reanalyzed the data in an ANOVA in which, besides the syntactic blocking (homogeneous vs. heterogeneous), we also included repetition (repeated vs. nonrepeated) as a factor. We found a main facilitatory effect of syntactic blocking, as before, $F_{1}(1,11)=5.4, M S E=4,410, p=$ $.042 ; F_{2}(1,35)=12.31, M S E=9,343, p=.001$, and a marginally significant $(13 \mathrm{~ms})$ inhibitory effect of repetition, $F_{1}(1,11)=4.7$, $M S E=1,842, p=.053 ; F_{2}(1,35)=3.74, M S E=4,052, p=.06$. The interaction between the two factors was not significant $\left(F_{1}\right.$ and $\left.F_{2}<1\right)$. Hence, the facilitatory effect we observed in the experiment cannot be accounted for in terms of repetition priming. ${ }^{4}$

\section{Experiment 3}

Having established in Experiments 1 and 2 that our paradigm allows us to assess the impact of semantic and syntactic factors in phrasal production, we move now to an experiment in which we crossed the two previous manipulations to assess whether the semantic and syntactic effects are additive or whether instead they interact. As described above, strictly serial models such as WEAVER $^{++}$would predict an additive effect of the two factors, whereas interactive models allow for the possibility of an interaction. In particular, if feedback is strong enough to affect lexicosemantic selection and if such interference effect is greater than the benefit derived from sharing the same syntactic frame, reaction times in the semantically and syntactically homogeneous condition should be slower than in any other condition.

\section{Method}

Participants. Eighteen Dutch native speakers from the participants pool of the Max Planck Institute for Psycholinguistics, none of whom had participated in Experiments 1 or 2, took part in this experiment and were each paid Hfl. 17.00 (approximately U.S. \$8). All participants were explicitly required to have good knowledge of English and filled out questionnaires regarding their level of English skills. The mean of the participants' self-reported level of English knowledge was $4.6(S D=0.6)$. The mean age at which these participants began to learn English was 10.6 years (range $=4-14$ ).

The words used in this experiment were of only slightly lower frequency to the ones used in Experiment 1 and 2 (see Materials section, below), but had a lower subjective familiarity to us. Therefore, participants were given a translation task before the experiment as a pretest. They were shown a list of all 36 English words used in this experiment and asked to provide a single-word Dutch translation. Six participants who gave incorrect responses for more than five translations (i.e., who scored less than $85 \%$ correct) were excluded from the experiment. Answers that were not identical to ones to be used in the study but were plausible translations and were of the same gender as the translation to be used in the study were counted as correct. For these plausible translations and for any other answers that participants did not know or that were clearly incorrect, we gave participants the correct answer and asked them to use this word in their responses in the study.

On completing this experiment, 3 participants reported using a strategy in which they began to pronounce the adjective immediately upon seeing the target. These participants were removed from the analysis and replaced, as were 2 participants who noted the blocking by gender.

Materials. For this experiment three semantic categories were used (animals, body parts, and weapons), with 12 exemplars each. These stimuli were chosen according to the same criteria as used in the previous studies. The English prompt words had an average word-form frequency of 75 per million in the CELEX database, whereas the Dutch response words had an average CELEX frequency of 82 per million. Within each category, six of the Dutch words were of the common gender and six were of the neuter gender. See Appendix B for a list of the stimuli.

\footnotetext{
${ }^{4}$ Yet another possible account for the effect in terms of repetition that does not require priming is that in the homogeneous condition the response set was half the size of the response set in the heterogeneous condition. Although we cannot evaluate this possibility because the different response set size is a characteristic built in our paradigm, this possibility seems to run counter to the fact that, if anything, our follow-up analysis suggests that repetition (which is by definition higher with fewer alternatives) may exert interference, not facilitation.
} 
From these 36 items, 24 lists of six items that were crossed with regards to semantic and gender homogeneity were created in the following manner. The 36 items were arranged in a $3 \times 12$ matrix such that rows corresponded to the three semantic categories and the left and the right half of the matrix (i.e., $3 \times 6$ items) corresponded to either grammatical gender. From this matrix, six possible semantically and syntactically homogeneous lists of six items can be derived. For the semantically homogeneous but syntactically heterogeneous condition, a further six lists were derived in which items were still from the same category but three were of common gender and the other three of neuter gender. For the semantically heterogeneous but syntactically homogeneous condition, lists consisted of pairs of items from the three semantic categories that were all from the same gender. Finally, for the semantically heterogeneous, syntactically heterogeneous sets, lists consisted of pairs of items from the three categories in which each member of a pair was of either gender.

From these 24 lists, experimental blocks were generated in which each of the six target words was presented six times, for a total of 36 trials per block. Within a block, each word was presented three times in each size. The presentation of words was pseudorandomized such that no target word appeared on consecutive trials and no more than three words of the same size appeared consecutively.

Design. The experimental design included semantic context (heterogeneous vs. homogeneous) and syntactic context (heterogeneous vs. homogeneous) as within-subjects variables.

Apparatus and procedure. These were identical to those used in Experiment 1. A practice block of 36 items was created for this experiment using six Dutch nouns stemming from three semantic categories and of mixed gender that were not used in the experimental trials.

The entire experiment consisted of 864 trials and lasted approximately $80 \mathrm{~min}$.

\section{Results and Discussion}

Responses judged to be incorrect by the experimenter, as well as response times longer than $1,500 \mathrm{~ms}$ or shorter that $250 \mathrm{~ms}$, were removed from the response time analysis $(8.5 \%)$. Again, the first presentation of each stimulus in each block was removed from the analysis.

Table 1 displays the mean response latencies and error percentages, varied by type of contextual blocking (semantic vs. syntactic) and context (heterogeneous vs. homogeneous). The response-time means indicate that both the semantic effect demonstrated in Experiment 1 and the syntactic effect obtained in Experiment 2 are replicated and that the two effects are clearly additive to one another.

An ANOVA was performed on the response-time data with semantic and syntactic variables as within-subjects factors. This analysis indicated a main effect of semantic context, $F_{1}(1$, $17)=18.51, M S E=4,428, p<.001 ; F_{2}(1,35)=21.81$, $M S E=8,679, p<.001$, as well as a main effect of syntactic context, $F_{1}(1,17)=18.82, M S E=5,148, p<.001 ; F_{2}(1$, $35)=17.70, M S E=11,856, p<.001$. Crucially, the interaction between semantic and syntactic context was not significant $\left(F_{1}=0.04, F_{2}<.01\right)$.

A second ANOVA, performed on the errors, indicated a significant main effect of semantic context, $F_{1}(1,17)=8.55$, $M S E=29.89, p=.009 ; F_{2}(1,35)=5.54, M S E=62.37, p=$ .024 , replicating the error findings from Experiment 1 . No significant effect of syntactic context was found $\left(F_{1}=0.87, F_{2}=2.54\right)$ replicating the null finding from Experiment 2. Consistent with the response-time analysis, no significant interaction between semantic and syntactic context was found $\left(F_{1}=1.36, F_{2}=2.03\right)$.
This experiment replicated the semantic interference effect reported in Experiment 1 and the syntactic congruency effect reported in Experiment 2; crucially, these two effects were additive. These results are compatible with models such as WEAVER ${ }^{++}$ but not with an interactive view that assumes that feedback is strong enough to affect lexico-semantic selection and in which the increased interference effect is greater than the benefit derived from sharing the same syntactic frame. ${ }^{5}$

\section{General Discussion}

By using a novel paradigm in which a translation task was combined with blocked presentation of items according to their semantic category and/or according to their gender, we have addressed questions concerning the interface between retrieving lexico-semantic and lexico-syntactic information in production. In the first experiment we found that response latencies to produce a phrase were longer for semantically homogeneous contexts than for semantically heterogeneous contexts. We interpret this finding in terms of lexical competition between semantically related items. In the second experiment, we found that response latencies were faster in gender-homogeneous contexts than in gender-heterogeneous contexts. This result can be interpreted in terms of facilitation in reaccessing a given syntactic property, and/or in reusing the same syntactic frame (Bock, 1986; Levelt et al., 1999). In our third experiment we found that when the semantic and gender conditions were crossed, response latencies were slower for semantically homogeneous contexts and faster for genderhomogeneous contexts, but crucially, these two factors did not interact.

\section{Considerations Concerning the Paradigm}

We introduced a novel paradigm to investigate lexical retrieval in production and we have shown that this paradigm allows us to tap into lexical and phrasal retrieval, providing converging evidence to previous chronometric studies that have used a pictureword interference task.

Whereas in this study we have shown that our paradigm that combines a translation task and a blocked design can effectively tap into phrasal production, in other experiments we have established that this task can be successfully used to investigate graded semantic effects for single-word production (Vigliocco et al., 2001). Furthermore, we also found that if picture naming is used instead of translation within a blocked design, we reliably observe semantic-interference effects (Damian et al., 2001).

With respect to single-word production, a similar paradigm has been used by Kroll and Stewart (1994) to investigate the organization of the bilingual lexicon. The authors reported that when a translation task was combined with a blocked design, asymmetries were present depending upon the direction (L1 to L2, or L2 to L1) of the required translation. In particular, Kroll and Stewart found a reliable semantic interference effect when bilingual DutchEnglish speakers were asked to translate blocks of homogeneous

\footnotetext{
${ }^{5}$ Note also that the lack of interaction we observed is a null effect in the context of relative small size main effects. Further research is therefore necessary to more firmly establish whether such a result may have arisen as a consequence of lack of statistical power in our study.
} 
and heterogeneous words from Dutch (L1) to English (L2), but not when the same task was performed from English (L2) to Dutch (L1). This result is prima facie inconsistent with our results, because we found a reliable semantic-interference effect in translation from English (L2) to Dutch (L1). It is important to note here that although our participants might have been somewhat more fluent speakers of English than the participants in Kroll and Stewart's experiment, in both cases speakers were not balanced bilinguals, but dominant Dutch bilinguals. Therefore the difference in results cannot be attributed to a difference in the investigated population. However, there is an important difference in the methodology between the two studies that can plausibly account for the different results. Namely, in Kroll and Stewart's experiment, participants were presented with each stimulus just once, whereas in our experiment each stimulus was repeated a number of times within and across blocks. Furthermore, in our experiment, each experimental block was preceded by the presentation of the corresponding translation pairs to allow participants to rehearse the translation for each item. These facts suggest that the involvement of lexico-semantics in translation depends on practice with the specific items, hence putative asymmetries in lexico-semantic involvement in processing by dominant bilingual speakers may reflect different amounts of practice with words in the second language. Because for dominant bilinguals different domains of knowledge may be more or less used in the second language, the likelihood of observing such asymmetries may depend on the domain of knowledge.

\section{The Representation and Retrieval of Lexico-Semantic and Lexico-Syntactic Information}

First, our experiments, within the domain of production processes, indicate that lexico-semantics and grammatical gender information are separable types of information as indicated by the fact that each of the two factors produced a main effect. This finding argues against a view such as the one proposed by Sera et al. (1994), according to which even arbitrary lexico-syntactic features such as grammatical gender, once learned, become part of the lexico-semantic specification of a word.

Second, our results provide some initial, novel information concerning the way in which activation flows between lexicosemantic and lexico-syntactic representations. Our results argue against a specific interactive activation system in which feedback from a syntactic frame is strong enough to affect lexico-semantic selection and the increase in interference due to the feedback is greater than the benefit derived from sharing the same syntactic frame. Hence, our results provide novel constraints that models will have to take into account. This is not, however, the only scenario that can be derived from interactive activation models. For example, it could be the case that the additive effect we observed is due to interactivity between lexico-semantic and lexico-syntactic processing. Suppose that the increased interference effect deriving from feedback in the lexico-semantic retrieval process is matched to a benefit in the lexico-syntactic retrieval process in the semantically and syntactically homogeneous blocks. That is, all the words included in such a block are semantically related, hence they are highly activated, hence they all send high amounts of activation to the same syntactic frame, increasing its activation to a higher extent than in the semantically heteroge- neous but syntactically homogeneous blocks in which the words, belonging to different semantic categories, are not as strongly activated. In this scenario, interactivity could lead to an additive effect in our experiment. We cannot discriminate between these two alternatives on the basis of the data at hand and hence a test of this possibility is left for future research. As indicated in the introduction, our study is among the very few that have, thus far, addressed the interface between the retrieval of lexico-semantic and lexico-syntactic information. Our findings are compatible with a strictly serial view such as proposed by Levelt et al. (1999).

Are the findings from the current study in line with prior evidence concerning the flow of activation between lexicosemantic and lexico-syntactic representations? In the introduction we discussed syntactic constraints on semantically related substitution errors as a type of evidence that can easily be accounted for, assuming feedback from syntactic frames to lexico-semantic representations, whereas these constraints present a problem for strictly serial views. Here, instead, we present data that are compatible with this latter type of models. Can we reconcile these findings? A recent study by Vigliocco, Vinson, Indefrey, and Levelt (2000) is relevant here. Vigliocco et al. (2000) assessed whether the gender constraint observed by Marx (1999) in spontaneously occurring semantic substitution errors in German can also be obtained in an experimental setting. They further introduced manipulations aimed at establishing how such a constraint arises. Errors were induced by asking participants to name blocks of pictures of objects from the same semantic categories presented one after the other on a computer screen. In one condition participants were asked to produce a definite-determiner + noun-phrase, and in another condition an indefinite-determiner + noun-phrase. Within each block, presenting objects from the same semantic category, words of different genders were represented and semantic errors were the dependent measure of interest. Regression analyses on the target-intruder confusion matrix were conducted in which both a measure of semantic similarity and the gender of the target and of the intruder were relevant predictors. The question addressed in the analyses was whether gender was a significant predictor above and beyond semantic similarity. It is important to note that when producing a definite-determiner + nounphrase, three different forms of the determiner are used, corresponding to the three genders in German (masculine, neuter, and feminine). However, when producing indefinite-determiner + noun-phrases only two forms of the determiner are used, because masculine and neuter nouns both take the same determiner (ein) whereas feminine nouns take a different determiner (eine). This different behavior of definite and indefinite determiner allowed Vigliocco et al. (2000) to assess whether the gender constraint observed in spontaneously occurring errors arises only as a consequence of feedback between lexico-semantic representations and syntactic frames or whether, instead, form dimensions mediate the effect. In particular, if the gender constraint arises only because of feedback between lexico-semantic representations and syntactic frames, we would predict to observe a gender constraint regardless of whether the determiner marks gender differentially. We found that the gender constraint was in fact mediated by the determiner used. That is, although we observed a gender constraint (namely, gender was found to be a significant predictor in the regression analysis above and beyond semantic similarity) for each gender when speakers produced definite-determiner + noun-phrases, no 
gender constraint was observed in the indefinite-determiner + noun-phrase condition for masculine and neuter nouns (for which the same determiner is used), but the constraint surfaced for feminine nouns (for which a different determiner is used). To present an account for these error results is beyond the scope of the current article. However, it is crucial to understand that these data cannot be interpreted solely on the basis of feedback from frames to lexical selection processes. Hence, the results from the error induction study and from the current study are not incompatible.

\section{The Relationship Between Lexico-Semantic and Lexico- Syntactic Information in Language Use}

Moving now from lexical retrieval in language production to language use in general, there are data concerning the relationship between lexico-semantic and gender information that seem to suggest a far more intimate relationship than what emerged in our investigation. For example, Konishi (1993) asked speakers of Spanish and speakers of German to rate words on the semantic differential scale (a strictly semantic task), and found that grammatically masculine words were rated higher on semantic dimensions that have masculine connotations, such as power and strength, and grammatically feminine words were rated higher on semantic dimensions that have feminine connotations, such as nurture. It is crucial to note that speakers of French and German differed in their ratings for words that had a different gender in the two languages (for example, the word sun is masculine in Spanish and feminine in German). On the basis of this correspondence between speakers' semantic ratings and the grammatical gender of nouns, Konishi argued that grammatical gender is intimately related to the conceptual representation for the words. Along similar lines, Sera et al. (1994) showed that grammatical gender is used in another semantic task, such as attributing a male or a female voice to a nonsexuated entity. In these studies it was found that although both younger (4-year-old) speakers of Spanish (a gender-marked language) and of English used a semantic criterion such as animacy in their attributions, older children and adult speakers of English continued to use animacy in their attributions, whereas speakers of Spanish used grammatical gender in addition. The authors interpreted these findings to support the nonseparability of lexico-semantic and gender information, once the latter is learned.

Thus, these observations suggest that syntactic properties such as gender can indeed affect semantics. The lack of interaction in our Experiment 3, instead, argues for the separability of these two types of information and seems in conflict with these other observations. However, the processes tapped in our study and in the above mentioned studies are very different. In our experiments we tapped into the automatic processes engaged online in sentence production whereas these other studies involved meta-cognitive semantic offline judgments. For these offline tasks, speakers may take advantage of the linguistic information about the gender of nouns as a further criterion on which to base their semantic judgments (see Bowers et al., 1999).

Our study suggests that the semantically driven lexical retrieval process is not influenced by the gender of the to-be-selected noun. Grammatical gender, however, is only one type of lexico-syntactic property, and it is a special type in that it is arguably only arbitrarily linked to semantic distinctions, whereas other lexicosyntactic properties have a higher degree of semantic motivation.
Hence, it is an empirical question to establish whether similar results can be obtained for other syntactic features with different degrees of semantic motivation.

\section{References}

Baayen, R. H., Piepenbrock, R., \& Gulikers, L. (1995). The CELEX lexical database (Release 2) [CD-ROM]. Philadelphia: Linguistic Data Consortium, University of Philadelphia.

Bates, E., \& MacWhinney, B. (1982). Functionalist approaches to grammar. In E. Wanner \& L. R. Gleitman (Eds.), Language acquisition: The state of the art (pp. 173-218). Cambridge, England: Cambridge University Press.

Berg, T. (1992). Productive and perceptual constraints on speech-error correction. Psychological Research, 54, 114-126.

Bock, J. K. (1986). Syntactic persistence in sentence production. Cognitive Psychology, 18, 355-387.

Bowers, J. S., Vigliocco, G., Stadthagen-Gonzales, H., \& Vinson, D. P. (1999). Distinguishing language from thought: Experimental evidence that syntax is lexically rather than conceptually represented. Psychological Science, 10, 310-315.

Buckingham, H. W. (1979). Linguistic aspects of lexical retrieval disturbances in the posterior fluent aphasics. In H. Whitaker \& H. A. Whitaker (Eds.), Studies in neurolinguistics (Vol. 4, pp. 269-290). New York: Academic Press.

Butterworth, B. (1989). Lexical access and representation in speech production. In W. Masler-Wilson (Ed.), Lexical representation and process (pp. 108-135). Cambridge, MA: MIT Press.

Caramazza, A. (1997). How many levels of processing are there in lexical access? Cognitive Neuropsychology, 14, 177-208.

Caramazza, A., \& Miozzo, M. (1997). The relation between syntactic and phonological knowledge in lexical access: Evidence from the "tip-ofthe-tongue" phenomenon. Cognition, 64, 309-343.

Damian, M. F., Vigliocco, G., Levelt, W. J. M. (2001). Effects of semantic context in the naming of pictures and words. Cognition, 81, B77-B86.

de Groot, A. M. B., Dannenburg, L., \& van Hell, J. G. (1994). Forward and backward word translation by bilinguals. Journal of Memory and Language, 33, 600-629.

Del Viso, S., Igoa, J. M., \& Garcia-Albea, J. H. (1987). Corpus of spontaneous slips of the tongue in Spanish. Unpublished work.

Dell, G. S. (1986). A spreading activation theory of retrieval in sentence production. Psychological Review, 93, 283-321.

Dell, G. S., Schwartz, M. F., Martin, N., Saffran, E. M., \& Gagnon, D. A. (1997). Lexical access in aphasic and nonaphasic speakers. Psychological Review, 104, 801-837.

Ferreira, V. (1996). Is it better to give than to donate? Syntactic flexibility in language production. Journal of Memory and Language, 35, 724755.

Foygel, D., \& Dell, G. S. (2000). Models of impaired lexical access in speech production. Journal of Memory and Language, 43, 182-216.

Fromkin, V. A. (1971). The non-anomalous nature of anomalous utterances. Language, 47, 27-52.

Garrett, M. F. (1975). The analysis of sentence production. In G. H. Bower (Ed.), The psychology of learning and motivation (pp. 133-177). New York: Academic Press.

Garrett, M. F. (1980). Levels of processing in sentence production. In B. Butterworth (Ed.), Language production: Vol. I. Speech and talk (pp. 177-221). London: Academic Press.

Garrett, M. F. (1982). Production of speech: Observations from normal and pathological language use. In A. W. Ellis (Ed.), Normality and pathology in cognitive functions (pp. 19-76). London: Academic Press.

Goodglass, H., Kaplan, E., Weintraub, S., \& Ackerman, N. (1976). The "tip-of-the-tongue" phenomenon in aphasia. Cortex, 12, 145-153.

Hartsuiker, R. J., \& Kolk, H. H. (1998). Syntactic facilitation in agrammatic sentence production. Brain and Language, 62, 221-254. 
Konishi, T. (1993). The semantics of grammatical gender: A cross-cultural study. Journal of Psycholinguistic Research, 22, 519-534.

Kroll, J., \& Stewart, E. (1994). Category interference in translation and picture naming: Evidence for asymmetric connections between bilingual memory representations. Journal of Memory and Language, 33, 149174

la Heij, W., de Bruyn, E., Elens, E., Hartsuiker, R., Helaha, D., \& van Schelven, L. (1990). Orthographic facilitation and categorical interference in a word-translation variant of the Stroop task. Canadian Journal of Psychology, 44, 76-83.

Langacker, R. W. (1987). Nouns and verbs. Language, 63, 53-94.

Levelt, W. J. M. (1989). Speaking: From intention to articulation. Cambridge, MA: MIT Press.

Levelt, W. J. M. (1999). Models of word production. Trends in Cognitive Science, 3, 223-232.

Levelt, W. J. M., Roelofs, A., \& Meyer, A. (1999). A theory of lexical access in speech production. Behavioral and Brain Sciences, 22, 1-75.

Marx, E. (1999). Gender processing in speech production: Evidence from German speech errors. Journal of Psycholinguistic Research, 28, 601621

McDonald, M. C., Pearlmutter, N. J., \& Seidenberg, M. S. (1994). The lexical nature of syntactic ambiguity resolution. Psychological Review, 101, 676-703.

Meyer, A. S. (1990). The time course of phonological encoding in language production: The encoding of successive syllables of a word. Journal of Memory and Language, 29, 524-545.

Miozzo, M., \& Caramazza, A. (1997). The retrieval of lexical-syntactic features. Journal of Experimental Psychology: Learning, Memory, and Cognition, 23, 1410-1423.

Miozzo, M., \& Caramazza, A. (1999). The selection of determiners in noun phrase production. Journal of Experimental Psychology: Learning, Memory, and Cognition, 25, 907-922.

Pickering, M. J., \& Branigan, H. P. (1999). Syntactic priming in language production. Trends in Cognitive Science, 3, 136-141.

Roelofs, A., \& Meyer, A. S. (1998). Metrical structure in planning the production of spoken words. Journal of Experimental Psychology: Learning, Memory, and Cognition, 24, 922-939.

Schelvis, M. (1985). The collection, categorization, storage, and retrieval of spontaneous speech error material at the Institute of Phonetics,
Utrecht. Utrecht, Netherlands: University of Utrecht, Institute of Phonetics.

Schriefers, H. (1993). Syntactic processes in the production of noun phrases. Journal of Experimental Psychology: Learning, Memory, and Cognition, 19, 841-850.

Schriefers, H., Meyer, A. S., \& Levelt, W. J. M. (1990). Exploring the time course of lexical access in language production: Picture-word interference studies. Journal of Memory and Language, 29, 86-102.

Schriefers, H., \& Teruel, E. (2000). Grammatical gender in noun phrase production: The gender interference effect in German. Journal of Experimental Psychology: Learning, Memory, and Cognition, 26, 1368 1377.

Sera, M., Berge, C. A. H., \& del Castillo-Pintado, J. (1994). Grammatical and conceptual forces in the attribution of gender by English and Spanish speakers. Cognitive Development, 9, 261-292.

Stemberger, J. P. (1985). An interactive activation model of language production. In A. Ellis (Ed.), Progress in the psychology of language (pp. 143-186). London: Erlbaum.

van Turennout, M., Hagoort, P., \& Brown, C. M. (1998, April 24). Brain activity during speaking: From syntax to phonology in 40 milliseconds. Science, 280, 572-574.

Vigliocco, G. (2001). Unpublished corpus of slips of the tongue in Italian. Unpublished work.

Vigliocco, G., Antonini, T., \& Garrett, M. F. (1997). Grammatical gender is on the tip of Italian tongues. Psychological Science, 8, 314-317.

Vigliocco, G., Damian, M. F., Lauer, \& Levelt, W. J. M. (2001). Graded semantic effects in naming. Manuscript in preparation.

Vigliocco, G., Vinson, D., Indefrey, P., \& Levelt, W. J. M. (2000, November). The interplay of meaning and syntax in speech: Insights from meaning related substitution errors. Paper presented at the 41st Annual Meeting of the Psychonomic Society, New Orleans, LA.

Vigliocco, G., Vinson, D. P., Martin, R., \& Garrett, M. F. (1999). Is "count" and "mass" information available when the noun is not? An investigation of tip of the tongue states and anomia. Journal of Memory and Language, 40, 534-558.

Vosse, T., \& Kempen, G. (2000). Syntactic structure assembly in human parsing: A computational model based on competitive inhibition and a lexicalist grammar. Cognition, 75, 105-143.

Appendix A

Stimuli (and Dutch Response Words) in Experiments 1 and 2

\begin{tabular}{|c|c|c|c|c|c|c|c|c|c|c|c|}
\hline \multicolumn{2}{|c|}{ Animals } & \multicolumn{2}{|c|}{ Body parts } & \multicolumn{2}{|c|}{ Clothing } & \multicolumn{2}{|c|}{ Plants } & \multicolumn{2}{|c|}{ Weapons } & \multicolumn{2}{|c|}{ Vehicles } \\
\hline English & Dutch & English & Dutch & English & Dutch & English & Dutch & English & Dutch & English & Dutch \\
\hline chicken & kip & tooth & tand & belt & riem & bush & struik & arrow & pijl & bicycle & fiets \\
\hline dog & hond & neck & hals & hat & hoed & flower & bloem & bullet & kogel & car & auto \\
\hline frog & kikker & muscle & spier & scarf & sjaal & vine & wijnstok & whip & zweep & ferry & pont \\
\hline horse & paard & & oog & shirt & hemd & leaf & blad & knife & mes & airplane & vliegtuig \\
\hline pig & varken & leg & been & suit & pak & weed & onkruid & sword & xwaard & raft & vlot \\
\hline rabbit & konijn & head & hoofd & underwear & ondergoed & forest & woud & rifle & geweer & ship & schip \\
\hline
\end{tabular}


Appendix B

Stimuli (and Dutch Response Words) in Experiment 3

\begin{tabular}{|c|c|c|c|c|c|}
\hline \multicolumn{2}{|c|}{ Animals } & \multicolumn{2}{|c|}{ Body parts } & \multicolumn{2}{|c|}{ Weapons } \\
\hline English & Dutch & English & Dutch & English & Dutch \\
\hline squirrel & eekhoorn & stomach & maag & lance & lans \\
\hline snake & slang & toe & teen & dagger & dolk \\
\hline monkey & aap & mouth & mond & missile & raket \\
\hline chicken & kip & wrist & pols & arrow & pijl \\
\hline $\operatorname{dog}$ & hond & tooth & tand & bullet & kogel \\
\hline frog & kikker & muscle & spier & whip & zweep \\
\hline horse & paard & eye & oog & knife & mes \\
\hline pig & varken & leg & been & sword & zwaard \\
\hline rabbit & konijn & head & hoofd & rifle & geweer \\
\hline sheep & schaap & bone & bot & pistol & pistool \\
\hline skunk & stinkdier & ear & oor & cannon & kanon \\
\hline deer & hert & heart & hart & pickaxe & houweel \\
\hline
\end{tabular}

Received August 17, 2000

\section{Low Publication Prices for APA Members and Affiliates}

Keeping you up-to-date. All APA Fellows, Members, Associates, and Student Affiliates receive-as part of their annual dues-subscriptions to the American Psychologist and APA Monitor. High School Teacher and International Affiliates receive subscriptions to the APA Monitor, and they may subscribe to the American Psychologist at a significantly reduced rate. In addition, all Members and Student Affiliates are eligible for savings of up to $60 \%$ (plus a journal credit) on all other APA journals, as well as significant discounts on subscriptions from cooperating societies and publishers (e.g., the American Association for Counseling and Development, Academic Press, and Human Sciences Press).

Essential resources. APA members and affiliates receive special rates for purchases of APA books, including the Publication Manual of the American Psychological Association, and on dozens of new topical books each year.

Other benefits of membership. Membership in APA also provides eligibility for competitive insurance plans, continuing education programs, reduced APA convention fees, and specialty divisions.

More information. Write to American Psychological Association, Membership Services, 750 First Street, NE, Washington, DC 20002-4242. 